\title{
KNOWLEDGE TRANSFER AND MANAGEMENT CONSULTING: A LOOK AT „THE FIRM”
}

In the authors' view, a firm's competitive advantage comes from the value it can develop for its customers. Most successful firms today can be considered ,intelligent enterprises” because they transform intellectual assets into product and service outputs. It follows that knowledge transfer is especially critical for the functioning of a management consulting firm, because knowledge is the cornerstone of the services such a firm offers its clients.

$\mathrm{W}$ hat is knowledge transfer (KT), and how does it relate to an organization? Why should a business such as a management consulting firm be concerned with it? Quinn (1992) maintains that most successful firms today can be considered "intelligent enterprises" because they transform intellectual assets from human input into product and service outputs. In both the service and manufacturing industries, most of the processes that contribute value to these outputs develop from knowledgebased activities (especially KT). Although KT is necessary for all organizations, it is especially critical for the functioning of a management consulting firm, because knowledge is the cornerstone of the services such a firm offers its clients.

Porter's (1985) work on organizational competitive advantage relates to this line of reasoning. He identified three general strategies for a company to establish an edge over its competitors in the marketplace:

- cost-providing products and/or services at the lowest price in the industry;

- differentiation-providing products and/or services uniquely related to a particular attribute (or multiple attributes) that customers value, which enables the firm to receive a premium dollar amount from its customers; and

u focus-meeting the needs of a select industry segment while omitting other segments.

A firm's competitive advantage comes from the value it can develop for its customers-value at emanates from the knowledge of its employees. As Tobin (1998) pointed out, employee knowledge is really what helps distinguish a firm from its competitors. Used via Porter's strategies, it determines a firm's competitive edge. This is specially true for a consulting firm, because its sustained success depends on the solutions its consultants provide to their clients. More specifically its sustained success is driven by he knowledge of its consultants, which is used to develop and deliver the service solutions to its clients. In discussing knowledge as well as how it is managed and transferred, we shall offer a case example of a consulting firm that relies on KT as an integral part of its functioning. In doing so, we aim
The process of managing and transferring knowledge effectively is the next big phase in the struggle for competitive advantage. to provide a starting point for addressing future issues related to KT, such as research and the refinement of consulting services.

\section{KNOWLEDGE}

$\Lambda$ number of philosophers, researchers, and practitioners Ahave attempted to define the concept of knowledge. Polanyi (1966) believed that knowledge, or the process of knowing, is personal and related to the individual. It can be viewed as a type of "intellectual capital" that has the ability to change how individuals and organizations view and create the world around them. Quinn, Anderson, and Finkelstein (1996) believe that the "intellect of an organization, in order of 
increasing importance, includes: (a) know what, (b) know how, (c) know why, and (d) care why." Liang (1994) has identified knowledge as one of four entities in a theoretical model of information processing. A data element is the most basic entity, because "conveys only a single value and is indivisible." Information is relevant data organized into a single message. Knowledge is created by combining related pieces of information over a period of time. Wisdom is the subset of new knowledge created when one uses one's deductive ability given a certain base of knowledge. In the taxonomy for this model, knowledge is not the same as information. Information relates to a message, while knowledge is developed and organized out of a procession of information based on the beliefs, values, and commitment of the individuals involved. Information becomes knowledge once it is understood and its value (including how it can be used) is learned. Therefore, information enables the creation of knowledge via learning.

From an epistemological perspective, human knowledge consists of two main types: explicit and tacit. Explicit knowledge can be codified. Although it is based on information, it is much richer because its value is understood. Explicit knowledge is conveyed through formal, methodical language. It is discrete and can be represented in books, archives, databases, and libraries.

In contrast, tacit or implicit knowledge is more subjectively related to individuals, making it difficult to formalize and transmit. Nonaka and Takeuchi (1995) contend that tacit knowledge is more of an ongoing process of understanding that is closely tied to "action, commitment, and involvement in a specific context" and involves viewpoints, intuition, and values that individuals develop through experience. Saint-Onge (1996) believes this type makes up the major part of organizational knowledge.

Tacit knowledge can be broken down into cognitive and technical elements. The cognitive element can be described by Johnson-Laird's (1983) mental models that allow individuals to create functional representations of their world by using analo-

Figure 1

The Knowledge Continuum

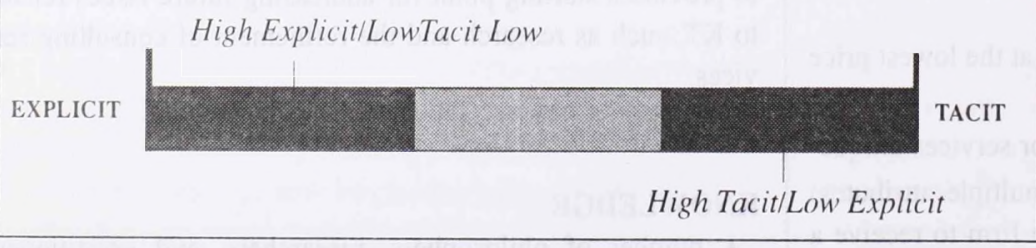

it is simpler to identify knowledge this way, we propose that this "dichotomy" needs to be qualified, because it does not really reflect the true nature of knowledge and KT. More specifically, knowledge may aetually exist on a continuum, with explicit knowledge and tacit knowledge anchoring the respective ends (see Figare 1). Our proposal is based on the assertion that some forms of explicit knowledge may be closer to tacit knowledge than other forms. For instance, a consulting methodology (a form of explicit knowledge) that gives a more detailed description of the organizational context and a variety of "if-then" scenarios offers a better indication of what should be done in a situation than a document that does not provide such a description.

Given the assumption of a knowledge continuum, the KT process for a certain situation is likely to involve a simultaneous transfer of degrees of tacit and explicit knowledge. However, for the sake of discussion, it is simpler to describe knowledge stemming more from the tacit end of the continuum (a high degree of tacit knowledge, a low degree of explicit knowledge) as tacit knowledge, and to describe that stemming more from the explicit end (high explicit, low tacit) as explicit knowledge. Thus, the use of the "dichotomy" should be a shortcut for referring to what is really a continuous relationship.

\section{KNOWLEDGE MANAGEMENT}

Zully understanding and appreciating KT involves consider$\mathrm{P}_{\text {ing it in a broader framework. Knowledge management }}$ (KM) has become not only a hot topic of conversation but, more important, a key focus in business and industry. With the service economy growing - of ten surpassing manufacturing each organization and its members need to use (transfer) knowledge as effectively as possible in providing its services. Generally, KM involves all the viewpoints and activities needed to comprehend, use, and benefit from capital in the form of organizational knowledge.

KM probably has its roots in a number of sources, but one key link is to information processing and theory, which relate to how data are handled or administered. Evolving from one medium, one sender, and one receiver, information processing has grown to involve individuals in more chaotic work situations, with myriad senders and receivers of information using multiple dissemination media. This growth of information processing and the subsequent development of information

gies in their minds. Examples include paradigms, schemata, and beliefs that assist people in understanding their environment. The technical element of tacit knowledge deals with definite skillls and know-how in a specific context.

Most work involving tacit and explicit knowledge implies that these components are dichotomous-that knowledge is considered either tacit or explicit, with no shades of gray. Although technology (IT) have resulted in what Porter and Millar (1985) called the "Information Revolution." In the mid-1980s, information and the related IT represented a competitive advantage, because they altered industry structure and the rules of competition, gave companies another way to outperform one another, and instigated the development of new businesses even from within existing operations. 
Merely gaining a competitive advantage is not enough, however; sustaining it really determines the long-term success of a company. With the ongoing expansion of an aggressive, global business market, it is harder for a company to distance itself from others because of the window of time associated with a competitive advantage-which starts when the company discovers and implements the advantage, and ends when competitors discover how to replicate it.

In the $1990 \mathrm{~s}$, most firms realized that information processing and technology were vital for business functioning. They have capitalized on this advantage by implementing IT solutions that allow them to produce, capture, analyze, and distribute more information that may or may not be of interest to the individuals who receive it. Having participated in the Information Revolution, they are now faced with the consequence of this advantage: information overload. Today's employees are often inundated with more information in more forms-reports, letters, e-mails, voice-mail, faxes, ad infinitumthan they have the time and capacity to comprehend and use effectively. From an organizational resource view-point, this overabundance of information is problematic because of the costly time and effort needed to identify which of the information can provide the most benefits and how to use it appropriately. This is a prime example of an ineffective use of organizational resources that ultimately affects company performance. Such an information overload helped provide the impetus for the development of KM.

KM and KT provide the means to take the mass of information resulting from the Information Revolution, understand its value, and determine how it can be made actionable-converted into knowledge so it can be used more appropriately. In addition to developing knowledge from information during work, employees can also "come in the door" with prior knowledge from previous work and other experience that also needs to be tapped proficiently. Responding to the need to use organizational resources effectively, KM and KT serve as enterprise-level means of separating knowledge from information, organizing it, and guiding its use to full capacity, thereby helping a firm distinguish itself from its competitors. Succinctly stated, KM and KT represent a present-day competitive advantage on which companies are trying to capitalize.

Like knowledge itself, $\mathrm{KM}$ is not a simple, one-dimensional construct. Consisting of many elements that can be described using a variety of nomenclature, it is best understood in terms of four key interrelated components: knowledge generation, knowledge representation, knowledge accessibility, and knowledge transfer.

\section{Knowledge Generation}

Knowledge generation involves activities that range from developing or creating new ideas and concepts, to identifying external knowledge and previously unnoticed trends, to integrating distinct concepts and practices. This ability of an organization as an open system to generate knowledge is due in part to what Cohen and Levinthal (1990) describe as its absorptive capacity They contend that the ability to assess and use knowledge is largely determined by the degree of prior related knowledge, which allows the capability to identify new and valuable information and understand how it can be tumed into knowledge.

A firm cannot create knowledge without people. Nonakaand Takeuchi propose that knowledge is generated through a cyclical or iterative transformation process of tacit and explicit knowledge involving individuals. Once the knowledge is developed, the firm integrates, magnifies, and solidifies it. Such knowledge does not necessarily come about in isolation; social interaction provides the setting for refining and validating the knowledge that is generated.

Through the iterative interaction between tacit and explicit knowledge, individual learning contributes to group knowledge, which in turn assists in the development of organizational knowledge. Nonaka and Takeuchi believe organizational learning is the conversion of explicit knowledge into tacit knowledge. While this description makes sense, the terms "organizational learning" and "knowledge management" must be distinguished from one another.

Organizational learning entails learning by individuals in a firm that becomes implanted in the structure, culture, and memory of that firm, allowing it to become more flexible and adaptive to its internal and external environments. Organizational learning is more than merely the sum of individual learning. As with the concept of gestalt, in which the whole is greater than the sum of its parts, there is some synergy among what individuals have learned that enables additional learning.

The concept of organizational learning also differs from a "learning organization." The latter is a company that continuously tries to become more adaptive and proactive in its environment by intentionally developing and using structures, processes, disciplines, and strategies to maximize what it learns as a whole. Organizational learning, on the other hand, is the action of using these structures, processes, disciplines, and strategies. In other words, a learning organization is what a firm wants to become, and organizational learning is how it achieves this goal.

Thus, the nature of the relationship between KM and organizational learning could really depend on how the two terms are defined. Nevertheless, they are not mutually exclusive concepts. They are iterative in nature. Organizational learning enables $\mathrm{KM}$, because it is through learning that the value and application of information is understood, thereby creating knowledge. KM also enables organizational learning, because the knowledge that is managed provides additional opportunities for learning that, in turn, can also create new knowledge. Moreover, formalizing the means and methods of learning helps create a learning organization. Knowledge management is a way of formalizing this learning through strategies, structures, and processes. 


\section{Knowledge Representation and Accessibility}

The second component of KM is knowledge representation, which entails comprehending what individuals know and turning it into a benefit or advantage for a firrn. This means translating the knowledge so that it can be conveyed in several waystraining modules, operation manuals, video presentations, expert-system software, and so on. Ultimately, this component serves to embed or implant the knowledge throughout the firm. Knowledge representation is virtually worthless unless the knowledge itself can be accessed. The key is to make represented organizational knowledge available to members of the firm. Knowledge can be accessed in a number of ways. For example, networks of people can be used to refer individuals to those who possess the needed expertise. Also, computer systems involving databases and search tools can be used to provide access to knowledge.

\section{Knowledge Transfer}

Finally, we come back to knowledge transfer, which is the fourth component of KM and arguably the most important. To enhance company performance, key knowledge must be able to be shared, disseminated, and used on a company-wide basis so that it becomes a potential asset. Knowledge transfer involves conveying and diffusing knowledge within a firm or among different firms. For example, an innovation in one department could be shared with or taught to another department or division of the same company. It could also be shared with a completely separate organization external to the company, such as when a consulting firm shares its expertise with its clients. Moreover, knowledge can be transferred from one firm to another without the first one's consent, such as when a competitor learns of and uses a key practice of the first firm.

Regular company communication, meetings, personal contacts, and training are among the ways in which knowledge can be conveyed. Explicit knowledge can be transferred through such media as books, archives, databases, and groupware technology. Tacit knowledge can best be transferred through personnel movement and the collaboration of individuals. This interaction allows them to understand and use the knowledge in a common context. Collaboration can be facilitated in many ways, ranging from on-the-job training (OJT) to job rotation to such structures as teams and cellular organizations (shifting of employees to satisfy a customer or company need).

To use KT appropriately, one must consider the type of knowledge (tacit or explicit) and the nature of the transfer (internal or external to the firm). Kogut and Zander (1993) discovered that more tacit or less codable knowledge is conveyed more easily and more frequently within a firm than outside it. Darr, Argote, and Epple (1995) found that more knowledge was transferred between affiliated organizations than between independent ones. These findings are due parrly to the fact that organizational members have a common context in which to interpret and adopt this relatively esoteric type of knowledge. Conversely, the more codable or explicit the knowledge is, the more it will be shared outside a firm. Because it is codable and can be documented in, say, a book or manual, it is easier for individuals outside a firm to understand and use.

\section{THE STUDY}

management consulting firm serves as an ideal site for Astudying the transfer of KT because KT is integral to its success. Such a firm must use knowledge to enhance its own functioning and provide products and services that increase the effectiveness of client companies. Consultants use a number of means to transfer knowledge, including technology such as groupware, documented methodologies, personnel movement, and collaboration. Studying the ways in which these means of transfer are used can assist in providing a better understanding of KT and serve as a basis for addressing future related issues, such as research and the refinement of consulting services.

Currently, the fields of KT and KM are in the early stages of development as a research area. In Snow and Thomas's (1994) classification, they fall somewhere between the description and explanation stages of theory-building, which involve identifying critical constructs, determining the relationships between them, and indicating the conceptual reasoning for them. These constructs and relationships are usually determined through methods that include observation and interviews.

Eisenhardt (1989) believed that an expert panel is especially suitable for new research areas that involve theory-building. Therefore, an expert panel of consultants was used to collect data for this preliminary study.

\section{Participants}

To ensure a substantial amount of expertise for the panel of consultants, individual position level and the number of years of relevant work experience were the key criteria used to select the members. A sample of six consultants four men and two women agreed to participate. They occupied senior manager, principal, or partner positions at a full-service, international consulting firm. Their consulting experience ranged from 8 to 21 years in duration, with a mean of 13.33 years. The length of time they had been employed by the firm ranged from 2 to 21 years, with a mean of 6.5 .

To provide a more representative sample of the firm, we selected each panel member from a different office of the firm's U.S. practice. Participants represented the Atlanta, Chicago, Los Angeles, New York, San Francisco, and St. Louis offices, although they did not necessarily work in the specific city nor with the same people on a given project. This diversity in locale helped minimize concerns over geographical biases.

\section{Procedures}

Because the nature of their work involves frequent travel and extended hours, all of the panel participants except one were interviewed by telephone. We used a structured interview format to obtain information, but if potential opportunities arose 
for gathering more information, we took advantage of those as well. With KM and KT in the early stages of development, any additional information could help provide a better understanding of the field.

In each interview, the respondent was asked 17 open-ended questions (listed in Figure 2) that addressed demographic, knowledge, and KT issues. Open-ended questions allowed the participants to respond of their own volition, free of the potential influence of preconceived answers. For questions that related to knowledge transfer, the participants were asked to think of a project or engagement they had worked on for at least six months in which knowledge was transferred successfully. This approach was taken to ensure that the participants were providing information based on their actual experience. The duration of the interviews averaged 60 minutes.

\section{Results}

The data obtained from participant interviews were analyzed for key themes. The interview items, the corresponding summarized themes, and supporting examples are as follows:

How do you define knowledge and knowledge transfer? In defining knowledge, the expert panel agreed with the research literature. One consultant encapsulated knowledge well, stating it was "processed information to which experience and value judgments have been added to make it more meaningful and usable." Panel members described KT as the diffusion and use of processed information, or the sharing of expertise to increase individual or organizational performance.

In what ways was knowledge transferred (on your project)? The panel reported that knowledge was transferred in a variety of ways. One consultant commented:

Technology is the most efficient way to ensure a broad transfer, because there is an immediate relationship between how the knowledge is created and how it is transferred.... [W]e produce a deliverable [work product] on computer so we don't have to transform the knowledge into another medium to transfer it.... K]nowledge related to a role can be transferred by training, mentoring, coaching, modeling, the buddy system, teamwork, and pairing, not just a transfer of material. ... [T] eaming is how knowledge is transferred within the firm.... I learned more in working with and through others.

Whether or not the participants realized it, the method of trans-

"Despite indica-

tions that all

knowledge could

be transferred.

the panel tended

to agree that the

most difficult kind

to transfer was

tacit.

\section{Figure 2}

\section{Interview Questions}

Demographic Information

1. What is your title?

2. How long have you worked in consulting?

3. How long have you worked for the current consulting firm?

4. In what city is your office located?

Knowledge Transfer

5. How do you define knowledge?

6. How do you define knowledge transfer?

For your responses to the following questions, think of a client project or engagement that you worked on for the consulting firm for a long period of time (at least six months) where knowledge was transferred successfully.

7. In what ways was knowledge transferred?

8. What influenced the transfer of knowledge?

9. Were there different types of knowledge that were transferred?

10. Were there any types of knowledge that could not be transferred?

11. Was knowledge transferred in any type of grouping?

12. How did the knowledge groupings differ from those that could not be transferred?

13. Was this knowledge transferred within the consulting firm, between the consulting firm and the client, or both?

14. What type of knowledge was transferred within the consulting firm?

15. What type of knowledge was transferred between the consulting firm and the client?

16. Does the transfer of knowledge within the consulting firm differ from that transferred between the consulting firm and the client?

17. Was there anything about the consulting firm's or the client's organizational structure that hindered the transfer of knowledge?

coaching, coun seling, modeling, storytelling, and learning-bydoing along with methods of collaboration involving teamwork, facilitated sessions, and a buddy system.

What influenced the transfer of knowledge? A number of factors influenced collaboration and the transfer of knowledge. One consultant from the expert panel listed three factors:

$\Leftrightarrow$ prior experience with or knowledge of the client project and consulting firm staff;

$\Rightarrow$ the career commitment level of the consultants and client personnel working on the project ("they learn what they have to leam just to finish [the project]"); and

$\Rightarrow$ whether evaluation will affect careers or finances ("the clients often do it because they have to").

The willingness or motivation of the individuals transferring knowledge, the capability of the transferor, and a common frame of reference were mentioned as influences on KT. Performance appraisals and the corresponding rewards also 
affected the willingness to collaborate and transfer knowledge. It was pointed out that individuals need to have a structured technique and must be accountable for transferring knowledge. The KT process could also be improved by structuring the knowledge in a format that reflects the recipients' preference for learning, as well as by including feedback to ensure that the transfer occurs.

Were different types of knowledge transferred? There was some discrepancy among the consultants on this question. One thought that the firm probably shares " 80 percent information and 20 percent knowledge"meaning perhaps that the information is in the process of being converted to knowledge based on the beliefs and commitment of individuals. The responses of the other consultants indicated that mainly explicit knowledge ("methodology of how to work together to determine leading practices for a given client situation and the technical specifications for products") and possibly some elements of tacit knowledge ("carrying on an interaction that is agreeable to the customer and doing so in a tone that showed customers were cared about") were transferred in their projects.

Were there types of knowledge that could not be transferred? The panel thought that, given enough time and motivation on the part of the transferor and recipient, most knowledge could be transferred. It was really a question of whether the cost of the transfer justified the benefit from the client's perspective. Despite indications that all knowledge could be transferred, the panel tended to agree that the most difficult kind to transfer was tacit ("experiential knowledge, managing complexity, intuition"), because it is "informal, unconscious, and unmanaged."

Was knowledge transferred in any type of grouping, and how did the groupings differ from those that could not be transferted? Knowledge was transferred in groupings, according to the panel members. This was done by sharing it in terms of the big picture and how it was to be applied. KT was enhanced by grouping knowledge into technical elements (such as "approaches and models") and nontechnical elements (such as "managing complexity"), which likely relate to explicit and tacit knowledge, respectively. Moreover, the firm established its own concept of a knowledge object (a "communication plan that represents milestones for getting information to people"), which is essentially a deliverable that is largely explicit in nature

The groupings that could be transferred differed from those that could not in two main ways. First, they were broader and more integrated, focusing on the knowledge in a more allencompassing manner. Second, they put the knowledge into context better so that individuals could see how it related to the organization. One consultant reported that knowledge should be grouped

in the context of how you make decisions to apply it knowledge of performance management, rating scales, link to pay, coaching and feedback, etc....
[T]ake all of the pieces and discuss them individually, but you need to group them together and talk about the big picture. .... [I]t doesn't add up unless you show what the context is.

What type of knowledge was transferred within the firm? The majority of the knowledge transferred within the firm (among consultants) as explicit (such as "technical knowledge, written methods, reengineering methodology"), which the firm appears to have done effectively. This finding supports the belief that explicit knowledge is easier to transfer because of its codability, being less context-specific than tacit knowledge. Tacit knowledge was transferred ("stories and feelings were combined with base data"), but with a lesser degree of effectiveness. The firm did not seem to do as good a job transferring knowledge internally via human interaction versus other methods of transfer.

What type of knowledge was transferred between the consulting firm and the client? Like knowledge that was transferred within the firm, the knowledge that was transferred to clients seemed to be a combination of explicit and tacit knowledge, and tended to be on the explicit end of the knowledge continuum. Examples of this were reengineering methods, technical knowledge (such as knowledge of SAP, an enterprise resource planning software), and business area knowledge, such as finance and HR. This type of knowledge was encodable and could be transferred via documentation and technology. The tacit knowledge did not necessarily seem to be transferred intentionally. Often, it would be a by-product of the process of collaboration and personnel movement. Examples included the transfer of base data with stories and feelings, as well as "elements of project management knowledge and change management knowledge."

Does the transfer of knowledge within the firm differ from that transferred between the firm and the client? "Protectionism" seemed to be an overriding theme in the transfer of knowledge from the consulting firm to the client, which related to the mutual benefits from the firm-client relationship. One consultant explained, "Knowledge is still power...so this causes one to be more cautious.... If you transfer too much knowledge to clients, they may not need you in the future." A key point made by one consultant was that the firm uses transfer media such as computers and books, which are more conducive to to sharing explicit knowledge. It did not seem to be as proficient at methods that could enhance the sharing of tacit knowledge, such as feedback and discussion.

Was there anything about the firm's or the client's organizational structure that hindered the transfer of knowledge? Structural hindrances identified by the expert panel included the reward and performance management systems and the composition of project teams. More specifically, there was a disconnection between performance and rewards for client personnel who were assigned to assist the consultants on projects. 
This was because the client personnel "reported to someone "Personal interaction and collaboration such as coaching. mentoring, OJT, and learning-by-doing should be used to transfer tacit knowledge effectively."

who was not on the project and had goals that had nothing to do with the project." Moreover, some personalities of project team members may have caused them to collaborate and communicate less openly, which decreased the effectiveness of the project teams. As one consultant suggested, this problem more than likely resulted from the project being "very topheavy, or having too many senior-level people working on it."

\section{BUSINESS IMPLICATIONS}

$\mathrm{T}_{\mathrm{o}}^{\mathrm{h}}$ he results of the interviews with the consulting firm's panel of experts suggest a number of implications for business and industry. Three key areas are particularly important to discuss regarding KT in practice: (a) explicit and tacit KT, (b) individual-level KT enablers and hindrances, and (c) organization-level KT enablers and hindrances. We will address each key area in more detail.

\section{Explicit and Tacit Knowledge Transfer}

Like many organizations, this consulting firm is in the early stages of trying to understand and use KT and KM better. Transfers of knowledge occur in a number of ways that relate to the type of knowledge involved. Granted, most companies do not expressly identify knowledge as being either tacit or explicit, but it is beneficial to understand the nature of what a company is trying to convey in order to accomplish KT more effectively. As mentioned earlier, this explicit and tacit distinction is really a shortcut for a continuous relationship between the two types, because varying degrees of tacit and explicit knowledge are likely to be transferred in a given situation. Nevertheless, being aware that certain types of knowledge are predominantly tacit or explicit helps determine how best to transfer them. Thus, some key points should be kept in mind regarding the types of knowledge involved in $\mathrm{KT}$ :

$\Rightarrow$ Documentation and technology can be used to transfer explicit knowledge effectively.

$\Rightarrow$ Personal interaction and collaboration such as coaching, mentoring, OJT, and learning-by-doing should be used to transfer tacit knowledge effectively.

$\Leftrightarrow$ A combination of these methods could be used (provided it is cost-effective) to ensure that both explicit and tacit knowledge related to a given issue are transferred.

\section{Individual-Level Knowledge Transfer \\ Enablers and Hindrances}

If both explicit and tacit KT are to occur, some conditions (enablers) related to individuals are necessary, and one hindrance needs to be diminished, if not eliminated: (1) the capa- bilities of the transferor and recipient, (2) the transferor/recipient willingness, (3) prior experience, (4) a common frame of reference, and (5) protectionism. Transferorswhether consultant or client-must first have the capacity to convey their knowledge to others. Then they must be willing to do so. In other words, they must not only possess the knowledge, but also be able and motivated to share it. Similarly, the recipients of the knowledge must be willing and able to listen and learn to ensure effective KT.

The element of prior experience entails the transferor and recipient each being aware of what the other has undergone prior to the transfer. What projects have they worked on? What industry experience do they possess? How effec-tive were any prior KT attempts in which they were involved? This is important, because the knowledge from these experiences will help determine what both parties see and use as the knowledge to be transferred. This enabler essentially addresses what is referred to as absorptive capacity.

Identifying and comprehending prior experience assists in establishing the fourth enabler: a common frame of reference. This is important to KT because it provides a shared understanding among participants so that the appropriate knowledge is soundly transferred. A note of caution: A common frame of reference should not be used to the point of excluding alternative perspectives that could initiate diversity and shed light on issues that otherwise may not have been addressed. Alternative perspectives can contribute to identifying new knowledge and determining how well the current knowledge is being transferred.

The hindrance that needs to be curtailed if not eliminated is protectionism, which can surface both within the firm and between the firm and its clients. A consulting firm is often perceived by consultants and clients as an organization of experts. Consequently, consultants may not want to share all of their knowledge with colleagues so they can maintain it as a kind of power that helps justify their status among colleagues. In working with clients, consultants may want to safeguard some of their knowledge in order to maintain an advantage in relation to those clients as well as other consulting firms.

Although this protectionism is undertaken to ensure the survival of the firm as well as the positions of the individuals within it, it needs to be kept in check because of the counterproductive effect it can have. The success of KT and ultimately KM depend heavily on both an internal and external environment that is conducive to sharing knowledge. If consultants are guarding and hoarding their knowledge to an extreme, the quality of the services their firm provides to both internal and external clients suffers. Other firms have the opportunity to capitalize on such poor performance as a result.

Key points to keep in mind regarding individual-level KT enablers and hindrances are:

$\Leftrightarrow$ Ensure that transferor and recipient have the appropriate capacity to transfer knowledge.

$\Leftrightarrow$ Minimize protectionism in reference to nonproprietary KT. 
$\rightarrow$ Enhance individual willingness and motivation to share knowledge.

$\rightarrow$ Be aware of each other's experience and try to establish a common frame of reference.

\section{Organization-level Knowledge Transfer Enablers and Hindrances}

While it is important to ensure that KT is encouraged at the individual level, it is just as importantif not more sothat it be supported at the level of the organization. The organization represents the enduring framework that can encourage collaboration and KT as well as the ultimate entity that benefits from them. Our case reveals two crucial organizational factors that can hinder KT (and could also enable it, if aptly used): systems and structures. More specifically, two critical systems the panel mentioned are performance management and compensation/benefits, which help formalize KT by reinforcing that the employer considers it important.

The performance management system is an area of concern because most firms do not yet hold their employees accountable for transferring knowledge. Individuals conducting performance appraisals may talk around KT, but they generally do not evaluate their direct reports based on how well the knowledge is transferred. One potential reason for this is that such evaluations are timeconsuming and often challenging. Our expert panel suggested that such issues as recipient preferences for acquiring knowledge and feedback on employee accomplishments should be involved. The panel also mentioned that the only way to ensure that KT has taken place successfully is to test for an understanding of the knowledge, or to observe employees' application of it on the job. The use of knowledge tests, behavioral observations, and/or multiple-source assessments is time-consuming, but the benefits should outweigh the costs. Evaluating the quality of KT comes down to making it a priority in the firm and reflecting this priority within the performance management system.

Our expert panel also revealed that a compensation/benefits system is an area of concern. Not only do employees need to be held accountable for KT in their work performance, they must also be rewarded for it, thereby increasing its quality and reliability in the future. If the reward system is not aligned with performance management, the quality of KT will be diminished and the performance management system will have less of an impact. Merely paying lip service to KT, identifying it as a phrase on performance appraisal forms, and giving occasional commendations for sharing knowledge are not enough. Consulting and client personnel should actually set objectives and be evaluated and rewarded based on how well the knowledge is transferred between them.

Ideally, other organizational structures or facets should be involved, such as the company form, job design, project work structure, office layout, company vision, strategy, and culture. More specifically, nontraditional and dynamic organizational forms, such as Miles and Snow's (1995) spherical form and
Miles, Snow, Mathews, Miles, and Coleman's (1997) cellular form, can be used to help shift individuals to address business needs. Jobs can be designed to incorporate more knowledgesharing. Formal and informal types of collaborative work structures (teams, communities of practice, social networks) can be used or encouraged. Office layouts that support knowledge sharing, such as a team room or open workspace environments, can enhance KT. Developing a knowledge vision for the firm and a strategy for reaching this vision would help incorporate and formalize KT and KM. Finally, the organizational culture should be conducive to knowledge sharing.

These examples do not represent an exhaustive list. But they should illustrate the point that KT and KM can be institutionalized within the firm in order to be productive for the long term and to be a true competitive advantage.

Key points to keep in mind regarding organization-level KT enablers and hindrances are:

$\rightarrow$ Refine compensation systems to provide an incentive for sharing knowledge.

$\rightarrow$ Revise performance management systems to make individuals accountable for transferring knowledge.

$\diamond$ Use organizational structures that encourage collaboration and KT.

$\rightarrow$ Develop a knowledge vision and strategy and align them with organizational systems to assist in developing a knowledge-sharing culture.

$\rightarrow$ Devise and implement the appropriate combination of knowledge repositories and informal knowledge transfer mechanisms (networks of individuals, peer coaching) in accordance with the knowledge strategy.

$\rightarrow$ Model knowledge-sharing behavior at the management level.

As management consulting firms and other companies strive A to establish and maintain a competitive advantage and use resources efficiently, knowledge management and its key component of knowledge transfer represent the next phase or means of doing so. KM and KT are more abstract and esoteric than most business concepts. Consequently, compa-nies need to be more open-minded and adopt another perspective to understand, appreciate, and benefit from them.

As Stewart (1995) pointed out, the effort of improving a firm's performance through the use of KM and KT is justified by the benefits: developing leading practices by transforming individual expertise into organizational knowledge; maintaining vigilance over competitors; and establishing a framework of intellectual resources that can help make the firm more efficient in terms of personnel and production. The key is that knowledge itself may not be subject to direct control, because it is intangible and based in the minds of organization members. But the process of managing and transferring it can be guided and supported to create an organizational asset.

Ultimately, it is only through institutionalizing KM and $\mathrm{KT}$, through various individual and organizational elements, on 
an enterprise-wide basis, that they will become a legitimate competitive advantage to such organizations as management consulting firms, as well as a benefit to those firms' clients.

\section{References}

W.M. Cohen and D.A. Levinthal, "Absorptive Capacity: A New Perspective on Learning and Innovation," Administrative Science Quarterly, March 1990, pp. 128- 152.

E.D. Darr, L. Argote, and D. Epple, "The Acquisition, Transfer, and Depreciation of Knowledge in Service Organizations: Productivity in Franchises," Management Science, November 1995, pp. 1,750-1,762.

K.M. Eisenhardt, "Building Theories from Case Study Research," Academy of Management Review, 14, 4 (1989): 532-550.

M.A. Gephart, V.I. Marsick, M.E. Van Buren, and M.S. Spiro, "Learning Organizations Come Alive," Training and Development, December 1996, pp. 34-45.

C. Gopal and J. Gagnon, "Five Steps to Better Knowledge Management," inset in "Knowledge, Information, Learning, and the IS Manager," Computerworld, June 1995 , p. 3.

P.N. Johnson-Laird, Mental Models (Cambridge, MA: Harvard University Press, 1983).

B. Kogut and U. Zander, "Knowledge of the Firm and the Evolutionary Theory of the Multinational Corporation," Journal of International Business Studies 24, 4 (1993): 625-645.

T. Liang, "The Basic Entity Model: A Fundamental Theoretical Model of Information and Information Processing," Information Processing and Management, 30, 5 (1994): 647-661.

R.E. Miles and C.C. Snow, "The New Network Firm: A Spherical Structure Built on a Human Investment Philosophy," Organizational Dynamics Spring 1995, pp. 5-18.

R.E. Miles, C.C. Snow, J.A. Mathews, G. Miles, and H.J. Coleman, "Organizing in the Knowledge Age: Anticipating the Cellular Form," Academy of Management Executive, November 1997, pp. 7-24.

I. Nonaka and H. Takeuchi, The Knowledge-Creating Company: How Japanese Companies Create the Dynamics of Innovation (New York: Oxford University Press, 1995).

M. Polanyi, The Tacit Dimension (London: Rutledge \& Kegan Paul, 1966).

M.E. Porter, Competitive Advantage: Creating and Sustaining Superior Performance (New York: Free Press, 1985).

M.E. Porter and V.E. Millar, "How Information Gives You Competitive Advantage," Harvard Business Review, JulyAugust 1985, pp. 149-160.
I.B. Quinn, "The Intelligent Enterprise: A New Paradigm," Academy of Management Executive, November 1992, pp. 48-63.

J.B. Quinn, P. Anderson, and S. Finkelstein, "Leveraging Intellect," Academy of Management Executive, August 1996, pp. 7-27.

B. Reimus, "Knowledge Sharing Within Management Consulting Firms: Reports on How U.S.-based Management Consultancies Deploy Technology, Use Groupware, and Facilitate Collaboration," (199C), Online: http://www.kennedypub.com/gware/html

H. Saint-Onge, "Tacit Knowledge: The Key to the Strategic Alignment of Intellectual Capital," Planning Review, 24, 2 (1996): 10-I4.

E.H. Schein, "Organizational Learning: What Is New? (1996), Online: http://leaming.mit.edu/res/wp/10012.html.

P.M. Senge, The Fifth Discipline: The Art and Practice of the Learning Organization (New York: Doubleday, 1990).

C.E. Shannon and W. Weaver; The Mathematical Theory of Communication (Urbana, IL: University of Illinois Press, 1949).

C.C. Snow and J.B. Thomas, "Field Research Methods in Strategic Management: Contributions to Theory Building and Testing," Journal of Management Studies July 1994, pp. 457-480.

T.A. Stewart, "Getting Real About Brainpower," Fortune, November 27, 1995, pp. 201-203.

K.E. Sveiby, "Transfer of Knowledge and the Information Processing Professions," European Management Journal, August 1996, pp. 379-388.

D.R. Tobin, The Knowledge-Enabled Organization: Moving from "Training" to Learning" to Meet Business Goals (New York: AMACOM, 1998).

N. Wiener, Cybernetics (Boston: MIT Press, 1948).

K.M. Wiig, Knowledge Management Foundations: Thinking About Thinking (Arlington, TX: Schema Press, 1993).

Ryan $K$. Lahti is a management consultant currently employed by the Hay Group's Organizational Effectiveness and Management Development practice in Los Angeles, California. Michael M. Beyerlein is the director of the Center for the Study of Work Teams and a professor of psychology at the University of North Texas.

The Editorial Board would like to express its gratitude to the authors for their kind permission to publish this article in our journal. 